\title{
Clinical outcomes of endoscopic ultrasound-guided ethanol injection for hepatocellular carcinoma in the caudate lobe
}

Authors

Institutions
So Nakaji ${ }^{1}$, Nobuto Hirata ${ }^{1}$, Rintaro Mikata ${ }^{2}$, Masayoshi Kobayashi ${ }^{1}$, Toshiyasu Shiratori ${ }^{1}$, Sadahisa Ogasawara ${ }^{2}$, Yoshihiko Ooka ${ }^{2}$, Toshio Tsuyuguchi ${ }^{2}$, Taketo Yamaguchi $^{3}$, Osamu Yokosuka ${ }^{2}$

${ }^{1}$ Department of Gastroenterology, Kameda Medical Center

2 Department of Gastroenterology and Nephrology, Graduate School of Medicine, Chiba University

${ }^{3}$ Department of Gastroenterology, Chiba Cancer Center submitted $\quad$ 6. April 2016 accepted after revision 19. August 2016

\section{Bibliography}

Dol http://dx.doi.org/ 10.1055/s-0042-116146 Published online: 30.9.2016 Endoscopy International Open 2016; 04: E1111-E1115

(c) Georg Thieme Verlag KG Stuttgart · New York E-ISSN 2196-9736

\section{Corresponding author}

\section{So Nakaji}

929, Higashi-cho,

Kamogawa City

Chiba, 296-8602

Japan

Fax: +81-4-7099-1131

nakajisou1978@yahoo.co.jp
Background and study aims: Accurately puncturing hepatocellular carcinomas (HCC) that arise from the caudate lobe is generally considered to be technically difficult. We conducted a retrospective study to evaluate the feasibility and safety (the therapeutic outcomes and adverse events) of endoscopic ultrasound (EUS)-guided ethanol injection as a novel treatment for HCC in the caudate lobe.

Patients and methods: Twelve patients with earlystage HCC of the caudate lobe that were treated with EUS-guided ethanol injection at two tertiary referral centers were reviewed retrospectively. To evaluate the therapeutic effect of the treatment, a local control curve and an overall

\section{Introduction \\ $\nabla$}

In general, surgical resection is considered to be curative for hepatocellular carcinoma (HCC). However, HCC that arises in the caudate lobe exhibits a higher recurrence rate after surgical resection than HCC that develops in other hepatic lobes. Moreover, isolated caudectomy is technically challenging, and it is also difficult to treat HCC in the caudate lobe using percutaneous local ablative therapy because ultrasonography often does not depict HCC clearly [1]. To solve this problem, we began performing endoscopic ultrasound (EUS)-guided ethanol injection as a treatment for such HCC. We conducted a retrospective study to evaluate the feasibility and safety (the therapeutic outcomes and adverse events) of EUS-guided ethanol injection as a novel treatment for HCC in the caudate lobe.

\section{Patients and methods $\nabla$}

The cases of patients with HCC in the caudate lobe that were treated with EUS-guided ethanol injection at 2 tertiary referral centers between Novem- survival curve were constructed using the Kaplan-Meier method.

Results: The mean follow-up duration was 31.0 months. The 1-year local control rate was $80.2 \%$, and recurrent lesions developed in 2 cases (after 3 and 9 months, respectively). The overall survival rate was $91.7 \%, 75.0 \%$, and $53.3 \%$ at 1,2 , and 3 years, respectively. Concerning procedurerelated adverse events (AEs), 2 patients suffered episodes of fever lasting a few days; however, no serious AEs occurred.

Conclusions: EUS-guided ethanol injection could be a useful treatment for early-stage HCC in the caudate lobe because of its simplicity and reduced invasiveness.

ber 2011 and November 2014 were reviewed. Eligibility criteria for treatment were as follows: (1) having $<3$ tumor foci, each measuring $\leq 3 \mathrm{~cm}$ in diameter; (2) a Child-Pugh score of $\leq 9$; (3) normal oral intake; (4) adequate major organ function levels; and (5) written informed consent. Exclusion criteria included anticoagulation therapy, active infections, serious psychiatric disease or dementia, multiple malignancies, and being allergic to alcohol.

The procedure was carried out under conscious sedation with monitoring of a patient's respiratory and circulatory dynamics. A curvilinear echoendoscope (GF-UCT240 or GF-UCT260; Olympus Medical Systems, Tokyo, Japan) and an ultrasound processor (ProSound F75 or ProSound SSD-5000SV; Hitachi Aloka Medical, Tokyo, Japan, or EU-ME1; Olympus Medical Systems) were used. The puncture procedure was performed using a 22-gauge or 25-gauge needle (Expect; Boston Scientific Corporation, Massachusetts, United States). In order to secure a safe ablative margin of $5 \mathrm{~mm}$ from the edge of the HCC, the maximum injected dose of ethanol was calculated based on the radius of the HCC using the following formula: $\left(4 \pi(\mathrm{r}+0.5)^{3} / 3\right)$. 

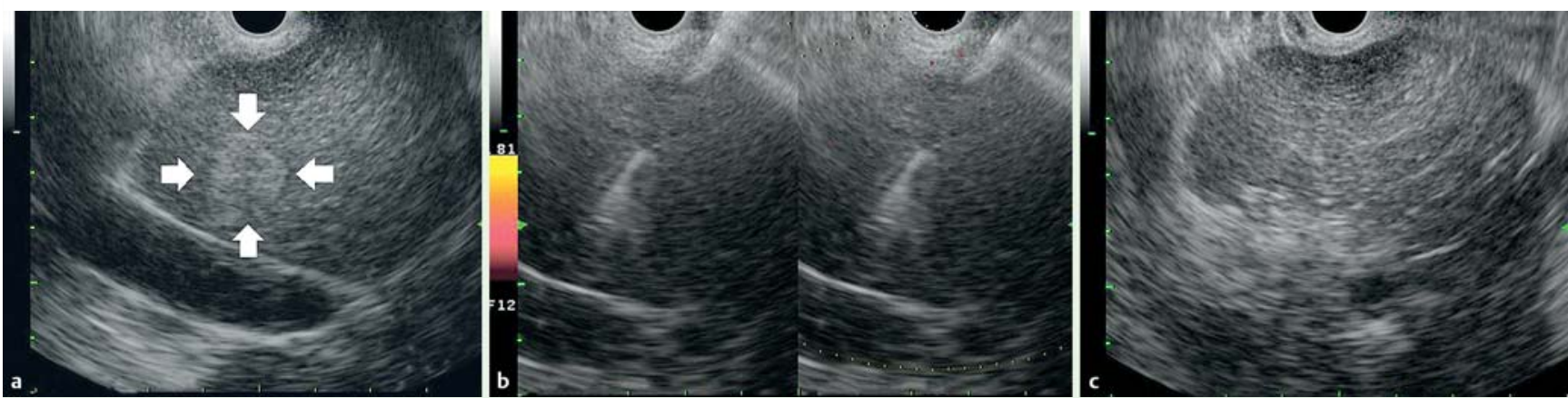

Fig. 1 a Hepatocellular carcinoma in the caudate lobe was visualized clearly on endoscopic ultrasound performed via the stomach. b Color Doppler ultrasound was obtained to ensure that the surrounding blood vessels were avoided, and a needle was inserted in the hepatocellular carcinoma. c After endoscopic ultrasound-guided ethanol injection, the hepatocellular carcinoma became hyperechoic.

Each dose was $\leq 10 \mathrm{~mL}$, and the procedures were performed twice or less a week ( $\bullet$ Fig. 1 ).

All patients were treated in hospital; laboratory tests were performed on the day of treatment or the following day to determine whether any procedure-related adverse events (AEs) had occurred; and imaging examinations; i.e., dynamic computed tomography (CT) or gadolinium ethoxybenzyl diethylenetriamine pentaacetic acid (Gd-EOB-DTPA)-enhanced magnetic resonance imaging (MRI), were conducted to assess whether the ethanol had been injected accurately. After being discharged, the patients visited an outpatient clinic at 3-month intervals during the first postoperative year. During these visits, the patients' alpha-fetoprotein and protein induced by vitamin $\mathrm{K}$ absence or antagonist-II (PIVKA-II) levels were examined, and dynamic CT or Gd-EOB-DTPA-MRI scans were carried out. Subsequently, the patients visited the outpatient clinic at predefined dates according to their condition.

To evaluate the therapeutic effect, a local control curve and an overall survival curve were constructed using the Kaplan-Meier method with EZR (Saitama Medical Center, Jichi Medical University, Saitama, Japan), which is a graphical user interface for $\mathrm{R}$ (The R Foundation for Statistical Computing, Vienna, Austria). More precisely, it is a modified version of $\mathrm{R}$ commander designed to add statistical functions frequently used in biostatistics [2]. This retrospective study was approved by the research ethics committee of Kameda Medical Center.

\section{Results}

$\nabla$

During the study period, 1349 patients were admitted with a diagnosis of HCC. In 35 of them, a HCC that for which local ablative therapy was indicated was located in the caudate lobe. Of these patients, 9 underwent transcatheter arterial chemoembolization (TACE) alone, 13 patients underwent radiofrequency ablation (RFA), and 12 patients underwent EUS-guided ethanol injection (4 patients underwent combination treatment involving EUSguided ethanol injection and TACE, and 8 patients underwent EUS-guided ethanol injection alone) as a treatment for HCC in the caudate lobe. The baseline characteristics of the EUS-guided ethanol injection group are summarized in Table 1, and the patients' characteristics associated with hepatocellular carcinoma are summarized in $\bullet$ Table 2.

The mean size of the HCC in the caudate lobe was $17.0 \mathrm{~mm}$, and the mean total injected dose of ethanol was $17.1 \mathrm{~mL}$. The mean duration of the follow-up period was 31.0 months ( $\bullet$ Table 3 ), and Fig. 2 shows a local control curve constructed using the Kaplan-Meier method. The 1-year local control rate was $80.2 \%$, and local recurrences occurred in Case 4 and Case 12. The intervals until recurrence in these cases were 3 months and 9 months, respectively. In Case 4, additional EUS-guided ethanol injections were performed to manage the locally recurrent lesion. The recurrent lesion was controlled for 33 months (from the additional treatment until the patient's death). On the other hand, in Case 12, CT-guided RFA was used to manage the locally recurrent HCC. The patients were divided into 2 groups; i.e., into those that did and did not receive combination treatment involving TACE ( $\checkmark$ Fig. 3); the 1-year local control rates of the combination therapy group and monotherapy group were $100 \%$ and $75.0 \%$, respectively. In addition, the patients were divided into those with and without new lesions ( $\bullet$ Fig. 4); the 1-year local control rates of the new lesion group and local recurrent group were $85.7 \%$, and $80.0 \%$, respectively. An overall survival curve is shown in Fig. 5. The overall survival rate was $91.7 \%, 75.0 \%$, and $53.3 \%$ at 1,2 , and 3 years, respectively. In Case 5, although the HCC in the caudate lobe was controlled, the patient died of multiple intrahepatic distant recurrent HCC only 8 months after treatment. Concerning procedure-related adverse events, 2 patients suffered episodes of fever lasting a few days; however, no serious AEs, e.g., intra-abdominal hemorrhaging, abscesses, or biloma, occurred.

\section{Discussion}

$\nabla$

Accurately puncturing HCC in the caudate lobe generally is considered difficult because of the distance from the body surface to the HCC. Therefore, HCC in the caudate lobe is often treated with TACE alone. However, Kim et al. reported local recurrence rates with TACE for HCC originating in the caudate lobe of $45 \%, 51 \%$, and $64 \%$ at 6 months, 1 year, and 3 years, respectively [3]. Concerning the local recurrence rate for percutaneous RFA, Hatanaka et al. reported that 1-year and 2-year local recurrence rates were both $12.5 \%$ [4], and Nishigaki et al. reported a 4-year local recurrence rate of $22.3 \%$ [5]. In terms of safety, no serious AEs occurred in previous studies [4-6]. However, it is conceivable that longdistance puncturing increases the incidence of local recurrence and AEs, at least in theory. On the other hand, in a study examining laparoscopic RFA Jiang et al. [7] reported that at 1, 2, 3, 4, and 5 years, overall survival rates were $96.3 \%, 88.9 \%, 74.1 \%, 74.1 \%$, and $62.9 \%$ and disease-free survival rates were $92.6 \%, 52.9 \%$, $44.4 \%, 33.3 \%$, and $33.3 \%$, respectively. Laparoscopic RFA has the 


\begin{tabular}{|cllllll|}
\hline Case & Age & Gender & PS & Underlying disease & Liver disease & Child-Pugh score \\
\hline 1 & 77 & M & 0 & HT & unknown & 5 \\
\hline 2 & 78 & M & 0 & HT & unknown & 5 \\
\hline 3 & 78 & M & 0 & HT & unknown & 5 \\
\hline 4 & 76 & M & 1 & DM/CVD & HCV & 5 \\
\hline 5 & 78 & M & 2 & DM/CVD & HCV & 7 \\
\hline 6 & 81 & M & 1 & HT/DM & HBV & 6 \\
\hline 7 & 64 & M & 0 & DM/HT & HCV & 5 \\
\hline 8 & 79 & M & 0 & HT & HCV & 5 \\
\hline 9 & 84 & M & 0 & HT & ALD & 5 \\
\hline 10 & 78 & M & 1 & none & HCV & 5 \\
\hline 11 & 67 & F & 1 & none & HCV & 6 \\
\hline 12 & 72 & F & 0 & HT/DM & unknown & 5 \\
\hline
\end{tabular}

Table 1 Patients' baseline characteristics.

PS, performance status; HCC, hepatocellular carcinoma; HT, hypertension; DM, diabetes mellitus; CVD, cerebrovascular disease; HBV, hepatitis B virus infection; HCV, hepatitis C virus infection; ALD, alcoholic liver disease.

\begin{tabular}{|cllllll|} 
Case & $\begin{array}{l}\text { Pathogenesis } \\
\text { of HCC in S1 }\end{array}$ & $\begin{array}{l}\text { Past treatments } \\
\text { for HCC in S1 }\end{array}$ & Location & $\begin{array}{l}\text { Number } \\
\text { of HCC }\end{array}$ & $\begin{array}{l}\text { Major axis } \\
\text { (mm) }\end{array}$ & $\begin{array}{l}\text { Combination } \\
\text { with TACE }\end{array}$ \\
\hline 1 & local recurrence & TACE & SL & 1 & 20 & + \\
\hline 2 & local recurrence & TACE & SL & 2 & 16 & + \\
\hline 3 & local recurrence & TACE & SL & 2 & 16 & + \\
\hline 4 & local recurrence & TACE & CP & 1 & 17 & - \\
\hline 5 & local recurrence & TACE & SL & 3 & 17 & - \\
\hline 6 & new & none & SL & 2 & 17 & - \\
\hline 7 & new & none & SL & 2 & 19 & - \\
\hline 8 & new & none & SL & 2 & 16 & - \\
\hline 9 & new & none & PCP & 3 & 11 & - \\
\hline 10 & new & none & CP & 2 & 13 & - \\
\hline 11 & new & none & SL & 3 & 25 & + \\
\hline 12 & new & none & SL & 1 & 20 & - \\
\hline
\end{tabular}

Table 2 Patients' characteristics associated with hepatocellular carcinoma.

HCC, hepatocellular carcinoma; TACE, transcatheter arterial chemoembolization; SL: Spiegel lobe; PCP, paracaval portion; CP, caudate process.

Table 3 Duration of follow-up period and outcomes.

\begin{tabular}{|c|c|c|c|}
\hline Case & $\begin{array}{l}\text { Time until local } \\
\text { recurrence (mo.) }\end{array}$ & $\begin{array}{l}\text { Follow-up period } \\
\text { (mo.) }\end{array}$ & Final outcome \\
\hline 1 & - & 51.1 & alive \\
\hline 2 & - & 41.7 & alive \\
\hline 3 & - & 41.7 & alive \\
\hline 4 & 3.4 & 35.5 & dead \\
\hline 5 & - & 10.6 & dead \\
\hline 6 & - & 17.0 & dead \\
\hline 7 & - & 36.9 & alive \\
\hline 8 & - & 20.8 & dead \\
\hline 9 & - & 32.9 & alive \\
\hline 10 & - & 32.6 & alive \\
\hline 11 & - & 24.1 & dead \\
\hline 12 & 8.5 & 27.4 & alive \\
\hline
\end{tabular}

advantage of improving puncture accuracy; however, it is invasive and inconvenient. By using EUS, we can easily visualize the caudate lobe in detail from the upper gastrointestinal tract. Consequently, HCC in the caudate lobe are easy to treat, even if they relapse locally, as was seen in Case 4.

Regarding whether it is necessary to perform TACE prior to ethanol injection, Fu et al. [8] reported that combination therapy involving TACE plus PEI exhibited better survival and recurrence rates than TACE or PEI alone. However, it is unclear whether such combination therapy is superior to monotherapy in cases involving HCC that measure $\leq 2 \mathrm{~cm}$ in diameter. In this study, 1 of 2 patients who developed recurrent disease underwent mono- therapy for a HCC that measured $17 \mathrm{~mm}$ in diameter; hence, combination therapy might be recommended for HCC in the caudate lobe, even in cases involving tumors smaller than $2 \mathrm{~cm}$ in diameter.

Recently, various EUS-based treatment methods for malignancies have been developed [9-11]. For example, EUS-guided neodymium:yttrium aluminum garnet (Nd:YAG) laser ablation has been described as a potential treatment for HCC [12]. On the other hand, we have reported the utility of EUS-guided ethanol injection in previous case reports $[13,14]$. To the best of our knowledge, this is the first case series to evaluate EUS-guided ethanol injection as a treatment for HCC in the caudate lobe. Compared with EUS-guided Nd:YAG laser ablation, EUS-guided ethanol injection has the advantage that it does not require a dedicated device. From a technical point of view, endoscopists must be careful to avoid collateral veins and esophageal varices during treatment. Therefore, screening esophagogastric duodenoscopy should be performed to check for varices, and if necessary, they should be dealt with before the procedure. Endoscopic injection sclerotherapy is superior to endoscopic variceal ligation because the collateral veins are treated simultaneously in the former procedure. During EUS-guided ethanol injection for HCC in the paracaval portion of the caudate lobe, the puncture route is restricted by the inferior vena cava and hepatic veins. For this reason, the local recurrence rate for HCC in the paracaval portion of the caudate lobe may be higher than that seen at other sites in the caudate lobe, e.g., the Spiegel lobe and caudate process.

Our study has some limitations. First, this was an uncontrolled retrospective study involving a small number of subjects. Second, 


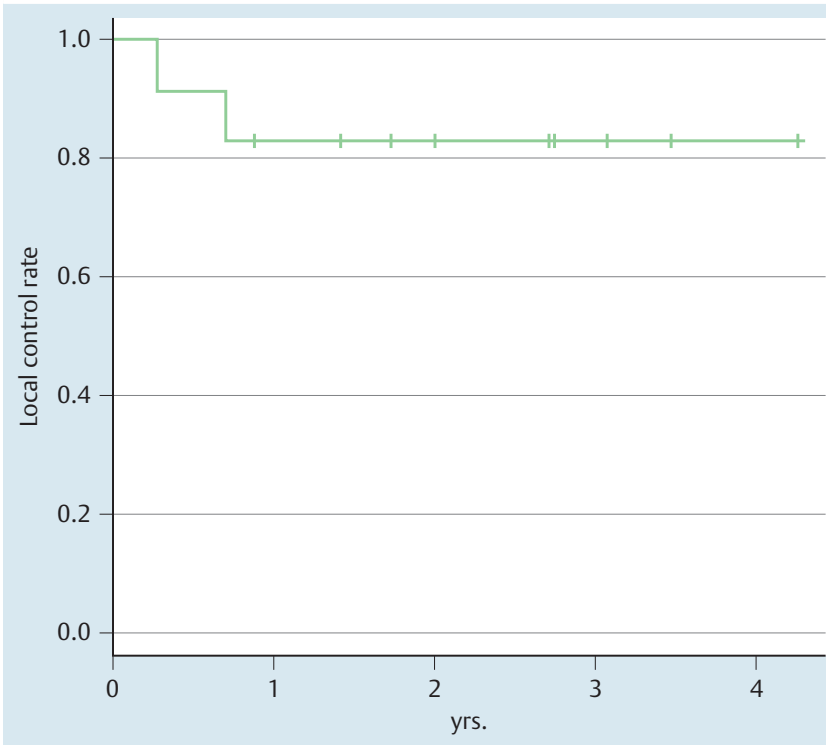

Fig. 2 Local tumor control curve constructed using the Kaplan-Meier method. The 1 -year local control rate was $80.2 \%$.

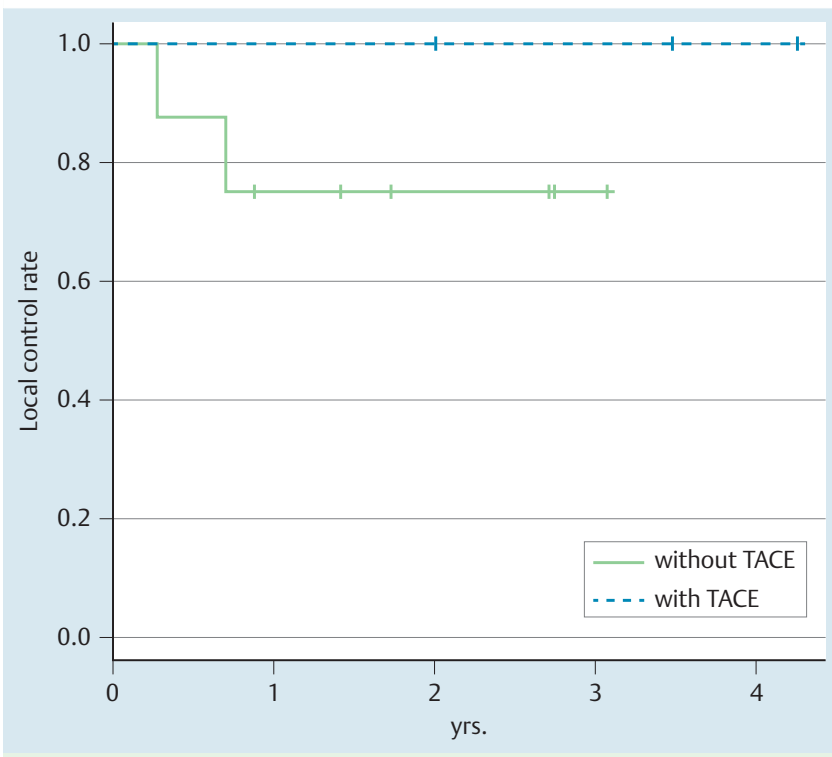

Fig. 3 Patients were divided into 2 groups; i. e., those who did and did not receive combination treatment involving TACE. One-year local control rates for the combination therapy group and monotherapy group were $100 \%$ and $75 \%$, respectively.

this study included cases of both primary and recurrent HCC in the caudate lobe. Moreover, not all of the patients were treated in the same way, e.g., some were treated with preceding TACE whereas others were not. Hence, further prospective studies are required to confirm the usefulness of EUS-guided ethanol injection for HCC in the caudate lobe.

\section{Conclusion}

\section{$\nabla$}

In conclusion, EUS-guided ethanol injection could become a useful treatment option for early-stage HCC in the caudate lobe because of its simplicity and reduced invasiveness.

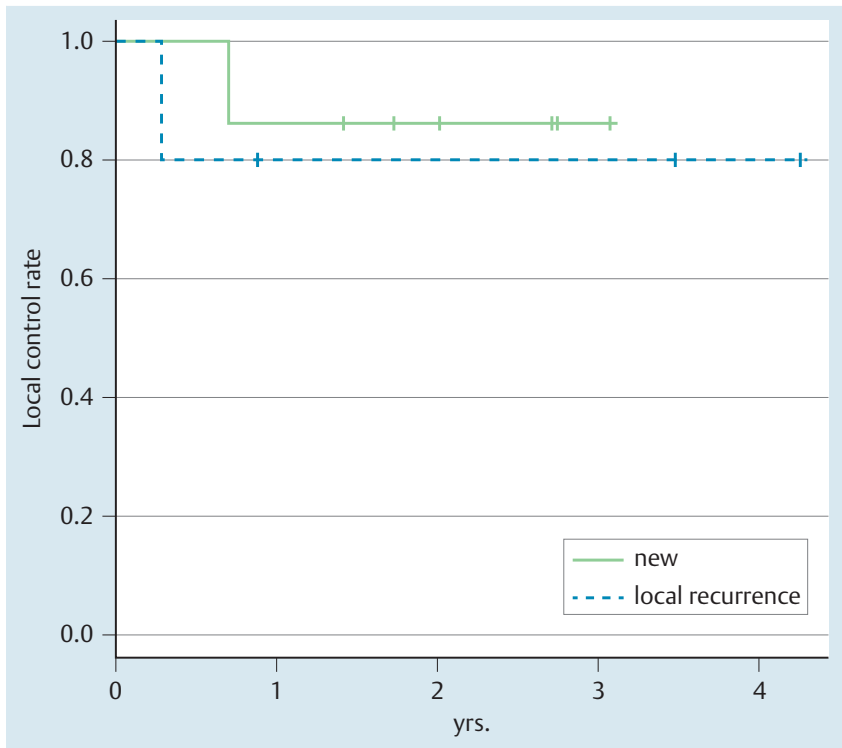

Fig.4 Patients were divided into those with and without new lesions. One-year local control rates for the new lesion group and local recurrence group were $85.7 \%$, and $80.0 \%$, respectively.

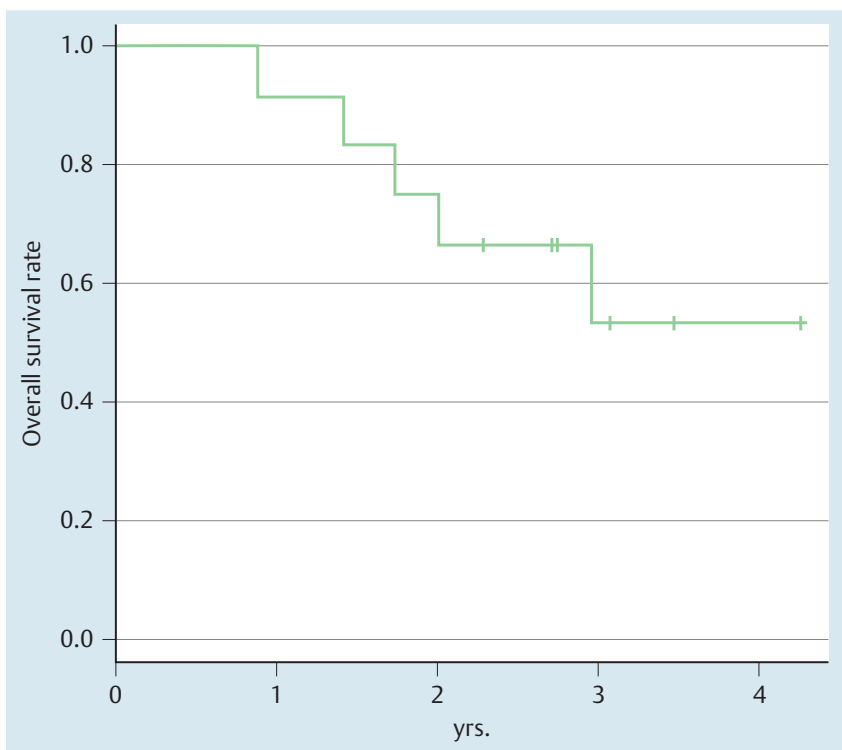

Fig. 5 The overall survival curve constructed using the Kaplan-Meier method. Overall survival rates were $91.7 \%, 75.0 \%$, and $53.3 \%$ at 1,2 , and 3 years, respectively.

\section{Competing interests: None}

\section{References}

1 Tanaka S, Shimada M, Shirabe K et al. Surgical outcome of patients with hepatocellular carcinoma originating in the caudate lobe. Am J Surg 2005; 190: $451-455$

2 Kanda $Y$. Investigation of the freely available easy-to-use software 'EZR' for medical statistics. Bone Marrow Transplant 2013; 48: 452 458

3 Kim HC, Chung JW, Jae HJ et al. Caudate lobe hepatocellular carcinoma treated with selective chemoembolization. Radiology 2010; 257: $278-287$

4 Hatanaka T, Kakizaki S, Yuhei S et al. Percutaneous radiofrequency ablation for hepatocellular carcinoma located in the caudate lobe of the liver. Acta Gastroenterol Belg 2015; 78: 267-273 
5 Nishigaki $Y$, Tomita E, Hayashi $H$ et al. Efficacy and safety of radiofrequency ablation for hepatocellular carcinoma in the caudate lobe of the liver. Hepatol Res 2013; 43: 467-474

6 Kariyama K, Nouso K, Wakuta A et al. Percutaneous radiofrequency ablation for treatment of hepatocellular carcinoma in the caudate lobe. Am J Roentgenol 2011; 197: W571-575

7 Jiang K, Zhang $W$, Su M et al. Laparoscopic radiofrequency ablation of solitary small hepatocellular carcinoma in the caudate lobe. Eur J Surg Oncol 2013; 39: 1236 - 1242

8 Fu Y, Zhao X, Yun Q et al. Transarterial chemoembolization (TACE) plus percutaneous ethanol injection (PEI) for the treatment of unresectable hepatocellular carcinoma: a meta-analysis of randomized controlled trials. Int J Clin Exp Med 2015; 8: 10388 - 10400

9 Gunter E, Lingenfelser T, Eitelbach $F$ et al. EUS-guided ethanol injection for treatment of a GI stromal tumor. Gastrointest Endosc 2003; 57: $113-115$
10 Jurgensen C, Schuppan D, Neser F et al. EUS-guided alcohol ablation of an insulinoma. Gastrointest Endosc 2006; 63: 1059-1062

11 Sun $S, X u H$, Xin $J$ et al. Endoscopic ultrasound-guided interstitial brachytherapy of unresectable pancreatic cancer: results of a pilot trial. Endoscopy 2006; 38: 399-403

12 Di Matteo F, Grasso R, Pacella CM et al. EUS-guided Nd:YAG laser ablation of a hepatocellular carcinoma in the caudate lobe. Gastrointest Endosc 2011; 73: 632-636

13 Nakaji S, Hirata $N$, Iwaki $K$ et al. Endoscopic ultrasound (EUS)-guided ethanol injection for hepatocellular carcinoma difficult to treat with percutaneous local treatment. Endoscopy 2012; 44 (Suppl. 02): E380

14 Nakaji S, Hirata $N$, Kobayashi $M$ et al. Endoscopic ultrasonographyguided ethanol injection as a treatment for ruptured hepatocellular carcinoma in the left hepatic lobe. Endoscopy 2015; 47 (Suppl. 01): E558-560 